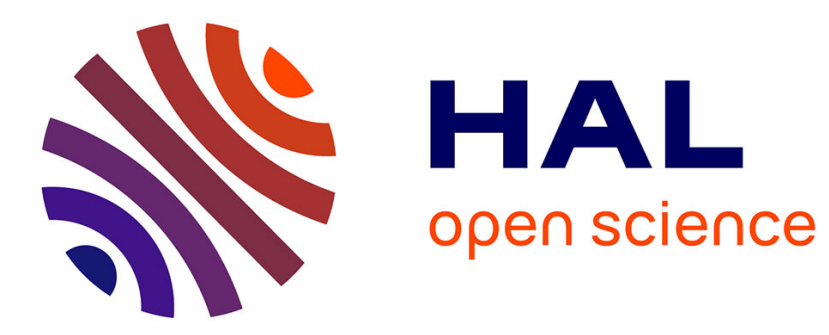

\title{
Assessing decision-making in elite academy footballers using real-world video clips
}

Javid Farahani, Pooya Soltani, Constantin Rezlescu

\section{To cite this version:}

Javid Farahani, Pooya Soltani, Constantin Rezlescu. Assessing decision-making in elite academy footballers using real-world video clips. Beth Parkin. Progress in brain research, 253, Elsevier, In press, 10.1016/bs.pbr.2020.06.015 . hal-02911917

\section{HAL Id: hal-02911917 https://hal.science/hal-02911917}

Submitted on 4 Aug 2020

HAL is a multi-disciplinary open access archive for the deposit and dissemination of scientific research documents, whether they are published or not. The documents may come from teaching and research institutions in France or abroad, or from public or private research centers.
L'archive ouverte pluridisciplinaire HAL, est destinée au dépôt et à la diffusion de documents scientifiques de niveau recherche, publiés ou non, émanant des établissements d'enseignement et de recherche français ou étrangers, des laboratoires publics ou privés. 


\section{PROGRESS IN BRAIN RESEARCH}

\section{Real-World Applications in Cognitive Neuroscience}

\section{3}

Farahani, J., Soltani, P., \& Rezlescu, C. (2020). Assessing decision-making in elite academy footballers using real-world video clips. Progress in Brain Research, 253. doi: $10.1016 /$ bs.pbr.2020.06.015

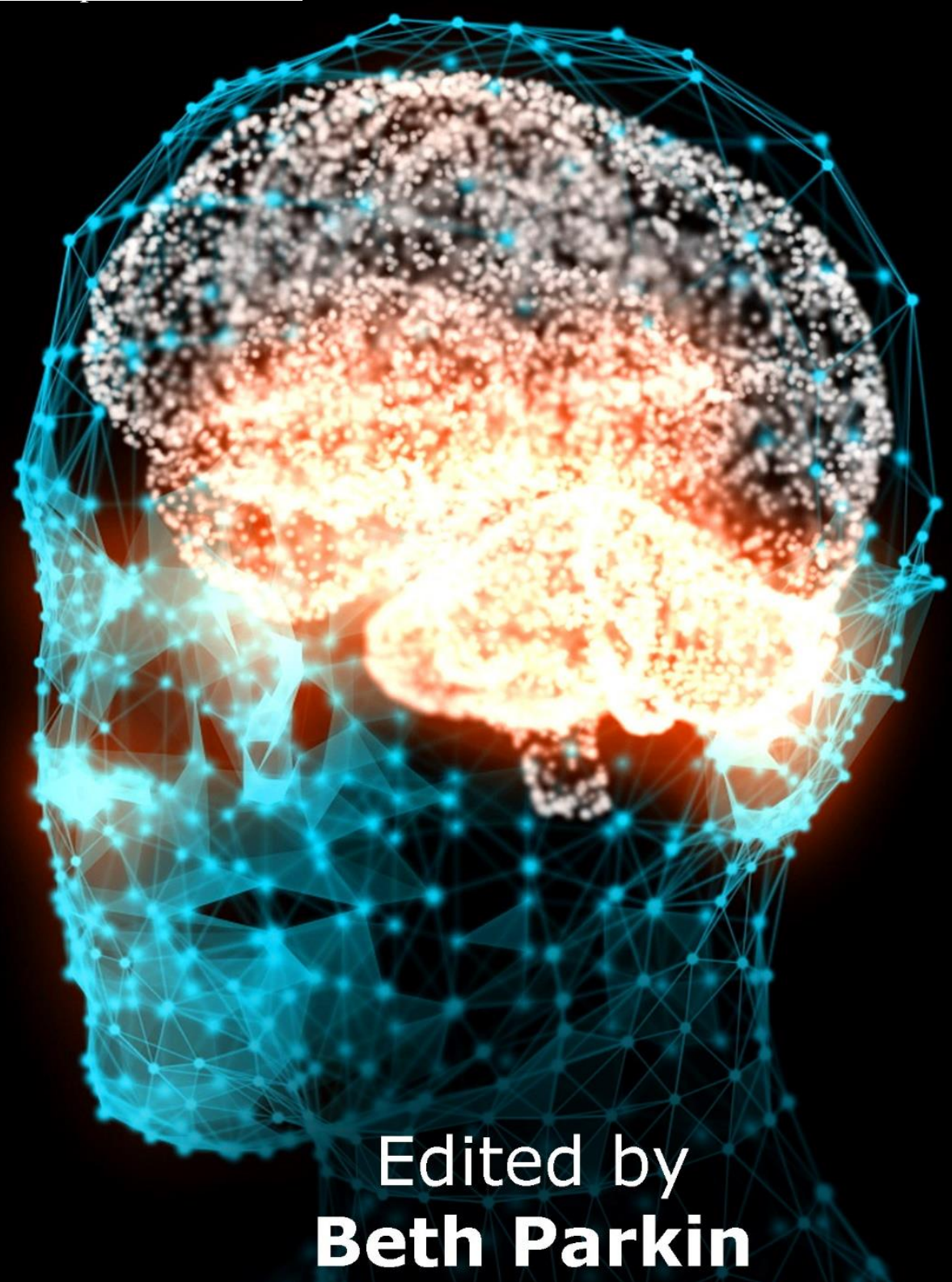




\title{
Assessing decision-making in elite academy footballers using real-world
}

\section{video clips}

\author{
Javid Farahani ${ }^{\mathrm{a}}$, Pooya Soltani ${ }^{\mathrm{b}, \mathrm{c}}$, Constantin Rezlescu ${ }^{\mathrm{d}}$ \\ ${ }^{a}$ Institute of Cognitive Neuroscience, University College London, Alexandra House, \\ Bloomsbury, London, United Kingdom \\ ${ }^{b}$ Centre for the Analysis of Motion, Entertainment Research and Applications (CAMERA), \\ Department of Computer Science, Department of Health, University of Bath, Bath, United \\ Kingdom \\ ${ }^{c}$ Department of Physical Education and Sport Sciences, School of Education and \\ Psychology, Shiraz University, Shiraz, Iran \\ ${ }^{d}$ Department of Experimental Psychology, University College London, London, United \\ Kingdom
}

\begin{abstract}
The aim of this experiment was to investigate whether there were differences in decision-making skills between different age groups (Under 16, 18 and 23) of elite academy footballers on a video-based task of real-life football scenarios. It also explored the relationship between individual performance on the task and the performance of the footballers on the pitch, as rated by three independent expert football coaches. This allowed us to examine whether this task is useful in predicting real-world decision-making skills. The results show that there was a significant difference in response times between response time was statistically significantly lower in U23 compared to U18 and U16 and there was no statistically significant difference between the U16 and the U18 groups, but no significant difference between age groups on the accuracy of response. The under 23 age group responded significantly quicker when compared to the under 18 and under 16 age group most quickly, then the U18, and finally, U16 footballers were the slowest on the task. In terms of comparing coaches' opinion about the players' decision-making skills and players performance on the task, there was a positive correlation between accuracy on the task and general decision-making skills rated by the coaches, suggesting that coaches have a good insight on what players can actually do as. However, coaches' ratings of decisionmaking skills and response times on the task did not correlate suggesting that coaches are not aware of the speed of decision-making, and that this is only measurable by a representative task.
\end{abstract}

Keywords: Expertise; Decision-making; Football; Elite athletes; Talent identification

\section{Introduction}

For young athletes, it is becoming increasingly difficult to break into Premier League first teams, as evidenced by the average age of starters in the Premier League increasing in recent years. In fact, less than $1 \%$ of Premier League academy footballers go on to make their debut at a professional level and more interestingly, $75 \%$ of those who do sign as professional academy footballers are no longer in the game by the time they are 21 years old. This is not only the case in English football but also in the Bundesliga (professional association football league in Germany) and La Liga (top professional football division of the Spanish football 
league system; FourFourTwo, 2018).

This situation means that identifying the right talents and helping young footballers to reach their maximum potential is the key to success at this level of competition for both elite academies and athletes. This is not only a challenge for academies and athletes, but researchers are also keen to understand what it takes to become an expert in a domain. In order to assess the progress of talent in elite academies, coaches and sports scientists consider different factors such as physical fitness (Carling et al., 2009; Meylan et al., 2010; Mujika et al., 2009), soccer-specific skills (Davids et al., 2000; Vaeyens et al., 2006), psychological traits (Morris, 2000; Murr et al., 2018a) and cognitive-perceptual skills (De Waelle et al., 2019; Huijgen et al., 2015). Recent studies on skill development in elite performance have shown that physical readiness of the athletes is not the single discriminating factor in their progress to the top level, rather, it has been shown that psychological and game intelligence attributes of elite athletes are also key factors in reaching peak performance (Ericsson et al., 2018). Consequently, over the last few years, there has been increasing interest in the psychological domain (Mann et al., 2007) in particular perceptual-cognitive skills (Murr et al., 2018b). In this paper, the focus is on the study of perceptual-cognitive skills in the final stage of the development of elite footballers, which starts around 15 years old and is completed by 23 years old.

Sports-relevant perceptual-cognitive skills (e.g., anticipation, pattern recognition, game intelligence and decision-making) comprise our ability to identify critical information from the environment and combine this information with existing knowledge of the domain to select and produce an appropriate response (Marteniuk, 1976). Among these skills, decision-making is considered the leading perceptual-cognitive skill involved in sport (Williams et al., 2004). Decision-making refers to the cognitive processes that underpin the selection of one course of action from several alternatives, and in the context of sport can be defined as the ability to perceive information, accurately interpret, then select an appropriate response (Baker et al., 2003).

In order to understand the mechanisms underlying perceptual-cognitive skills, notable research has been conducted during the last three decades, and the study of expertise across different domains and professions has now become a well-established domain in sports science and cognitive psychology (e.g., Aglioti et al., 2008, Ericsson, 1996; Roberts et al., 2010, Roberts et al., 2012; Starkes and Ericsson, 2003).

One of the most established theoretical frameworks used to study elite performance was developed by Ericsson and Smith (1991). They introduced a theoretical framework to understand how elite individuals function in different domains, called the elite performance approach (EPA). This approach includes three stages in the study of expertise. The first stage is to capture elite performance through testing in the laboratory. The second stage is to use process-tracing methods such as eye movement, film occlusion and verbal reports to distinguish elite performers from novices, and the final stage of the EPA is to examine the acquisition of the identified characteristics of expertise.

The most common method used in stage one of the EPA in elite performance is the expert-novice paradigm for identifying the elite performers. Field or laboratory testing is used to elicit the differences in expert and novice performance. Results to date show that elite athletes benefit from superior perceptual-cognitive skills, such as anticipation (Abernethy et al., 1994; Williams et al., 2003), pattern recall and recognition (Abernethy et al., 2005, Gilis et al., 2008, Baker et al., 2003), and decision- making (Abernethy and Chua, 1996; Lorains et al., 2013; Starkes and Lindley, 1994). Among these skills, MacMahon and McPherson (2009) suggested that decision-making is the primary skill and pattern recall and recognition and anticipation are secondary factors, contributing to elite performance. 
Research that examines decision-making in sport has shown that it can be a complex skill to capture in a laboratory, particularly using video-based tasks (e.g., MacMahon et al., 2007). Due to the complex nature of the perceptual-cognitive skills in a competitive football match, designing task representative methods that comprise the entire decision-making process, particularly using video-based tasks (MacMahon et al., 2007), is extremely challenging (De Waelle et al., 2019). With recent advances in video technologies, scientists are now able to use video-based technology to develop task representative decision-making assessment. Video-based assessment for decision-making in sport usually refers to watching a series of short clips, between 5 and $15 \mathrm{~s}$ and making a decision on a specific scenario via a mouse-click or tapping on a touch-screen (Lorains et al., 2013).

This approach is widely used to study handball (Johnson and Raab, 2003, Salimin et al., 2015; Tallir et al., 2003a, Tallir et al., 2003b), Basketball (Hepler and Feltz, 2012; Tallir et al., 2003a, Tallir et al., 2003b), Australian Rugby (Buszard et al., 2013), junior Australian football players talent identification (Makdissi and Davis, 2016; Woods et al., 2016), youth soccer (De Waelle et al., 2019) and performance of sporting officials (Elsworthy et al., 2014; Kittel et al., 2019; MacMahon et al., 2007). The results show that video-based assessment for decision-making should be considered a reliable method to differentiate elite and novice athletes. Also, it has been suggested that the benefits of video-based assessment are the ability to control the environment and conditions for all players in a way that is not possible in the field (Berry et al., 2008; Bruce et al., 2012).

In an early attempt to use video-based simulation in decision-making in sport, Johnson and Raab (2003) tested elite handball players on a video-based task and asked them to make their decision as soon as possible. They found that when players chose the first option they generated, they were more accurate. This finding was supported by the later research of Hepler and Feltz (2012) as they also found higher quality in a decision-making task when there is time pressure to make a one-shot choice.

Lorains et al. (2013), used video-based assessment in Australian football and compared elite, sub-elite and novice players on different video speeds. They tested athletes on seven different speed multipliers for playing the video $(0.5,0.75,1.1 .25,1.5,1.75$ and 2 $x)$ and asked them to choose the best option for each scenario. They reported superior performance on the task for the elite players when they were tested in above real-time (faster speed on video than normal). Also, their result showed that elite and sub-elite players perceived $1.25 \times$ speed for the video as the most game-like feeling.

More recently, Weigel et al. (2015) employed video-based assessment to investigate the visual strategy of the soccer players. The work compared elite players to novices with no high-level handball experience. They also reported that elite players are faster in processing information than novices on the task, which may help them to use the extra time for judgment and therefore achieve improved accuracy.

Another study that used video-based assessment, Woods et al. (2016), used this methodology to discriminate junior talented Australian footballers from their non-talented counterparts. Participants completed a video-based task which included scenarios from Australian football. After watching each clip, participants recorded their answer for choosing the best passing option by drawing a circle on a paper representing their choice. Due to the low technology design of this experiment, no response time is captured but decision-making time was limited to $3 \mathrm{~s}$. Woods et al. (2016) reported a significant effect between groups and therefore suggested that their method is able to identify talented players from non-talented ones.

Recently, scientists used the video-based assessment methods to explore perceptualcognitive skills among soccer players. Wirth et al. (2018) compared elite soccer players with novices on a 360-degree video environment on real-life scenarios. As they used virtual reality 
headsets instead of a computer or tablet-based platforms, it is not possible to compare their results in terms of response time with the previous studies, however, they report quicker response time for elite players in comparison to novice counterparts in this specific task.

A recent study using video assessment paradigms was conducted by De Waelle et al. (2019) in youth soccer. To our knowledge, their sample size (328 academy youth soccer players) is the largest sample in this domain. They employed players from tier 1 (late childhood), tier 2 (early adolescence) and tier 3 (mid-adolescence) of the Belgian football league and a control group of youth athletes with no experience of soccer. In terms of the design of the experiment, participants were asked to make decisions on clips on 2 vs 1,3 vs 1,3 vs 2,4 vs 3 and 5 vs 3 scenarios. These numbers refer to the number of players. De Waelle et al. (2019) reported that youth soccer players were more accurate than the control group. They concluded that coaches and clubs must be aware of the limits of approach and data interpretation, as there is only limited supporting evidence for the usefulness of videobased assessment in youth soccer.

In the current study we examine the development of decision-making skills in elite academy soccer. The primary objective is to investigate the differences between Under 16, 18 and 23 elite academy soccer players on a video-based decision-making assessment. The second objective is to compare participants' performance on the task in terms of accuracy and response time with their performance on the pitch as measured by coaches' opinions. To our knowledge, this one is the very first attempt to understand decision-making skills development in elite academy soccer players and little is known about the accuracy of coaches' opinions and the player's performance on a video-based representative task.

\section{Methods and materials}

\section{Participants}

In total, 73 male participants (range 15-23 years old, mean 18.6 years old) took part in three groups (U16, U18 and U23). The U23 group comprised 26 footballers with a mean age of 21.4 ( $\mathrm{SD}=1.02)$ who had been training at the Premier League academy level for at least 5 years. The U18 participants were 24 Premier League academy footballers with a mean age of $17.8(\mathrm{SD}=0.5)$ and at least 4 years of experience at the Premier League level. The U16 group were 23 Premier League academy footballers with a mean age of 15.9 years $(\mathrm{SD}=0.38)$ and a minimum of 4 years of experience at Premier League academy level. They were all based at one of two major Premier League football academies. All participants had a normal or corrected-to-normal vision. All participants gave their written informed consent in accordance with the Declaration of Helsinki and the guidelines approved by the Ethical Committee of University College London (UCL).

\section{Experimental design}

The study comprised of three experimental groups which differed in age and experience. Each participant performed the tasks while seated using an individual tablet in a quiet room. All testing sessions happened in the morning after team breakfast and before the training session to prevent the effect of physical fatigue due to exercise. Participants were asked to perform a tablet-based task by watching short video clips of football matches.

The task consisted of two parts. First, there was a practice session (comprised of 5 trials), followed by the task (which consisted of 20 trials). The participants were first shown a paused video of a football match with the location of the ball highlighted for $2 \mathrm{~s}$. The video 
clip then played for $5 \mathrm{~s}$, after which the screen paused again and three locations where the ball could have been passed to were highlighted (see Fig. 1). The participants' task was to indicate the best option in terms of building a good attacking scenario by tapping on their choice of location on the tablet screen. They had $4 \mathrm{~s}$ to make a decision, after which the software moved to the next clips. Options were rated indicating the best choice (value $=1$ ), an intermediate choice (value $=2$ ), and the worst choice (value $=3$ ), no response was categorized as a value 3 decision. To determine the best choices for each scenario, each video clip was watched and independently rated by three UEFA (Union of European Football Associations) license coaches and only clips on which two or all three raters agreed were used.

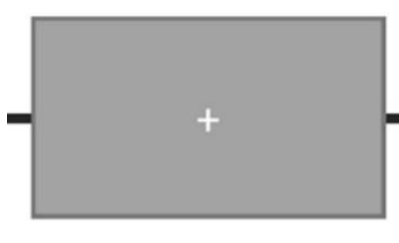

$1 \mathrm{~s}$ Fixation Cross

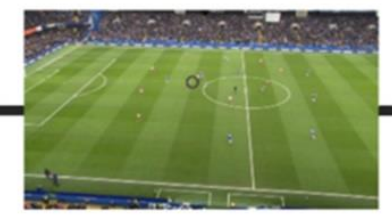

$1 \mathrm{~s}$ First Frame

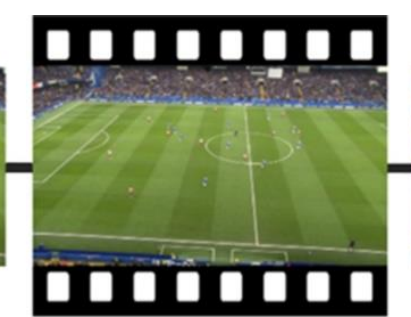

$5 \mathrm{~s}$

Clip

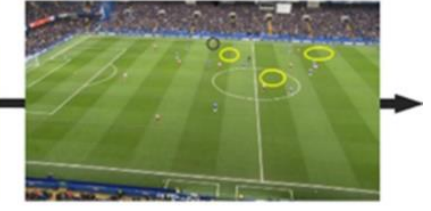

$4 \mathrm{~s}$

Response

Fig. 1. Diagram showing the order and duration of stimuli for one trial.

The video clips were extracted from Premier League matches by a camera placed in the middle of the field. The duration of the video clips were $5 \mathrm{~s}$ and no feedback was provided. Trials were separated by a fixation cross on the screen for $1 \mathrm{~s}$. Participants were asked to respond as quickly and as accurately as possible. Accuracy on the task was measured by converting the value 1-3 to the percentage of the maximum possible score, and response time was measured in milliseconds. Fig. 1 shows sample screens of one trial.

The experiment was developed on the Game Intuition ${ }^{\circledR}$ platform (London Cognition limited, UK) using the web portal for editing the videos and building the decision-making screen. An iOS tablet pro with 10.5 in. touch (made by Apple Inc., USA) screen was used for the presentation of stimuli and participants were required to tap on the screen of the tablet to indicate their responses.

In order to compare the performance of the participants on the task with how their coaches rated them on the pitch in terms of the judgment of their decision-making skills, a Likert scale of 1 to 10 was used, where 1 represented the lowest and 10 the highest quality. Coaches rated each player on four specific qualities. The first was general decision-making (GeneralDM) ability in the game including attacking and defending scenarios, this covers all the types of decisions a player needs to make in a match. The second was on attacking decision-making skills (AttackingDM), which means when a player's team has possession and are going to attack the opponent's goal. This includes the decisions made by players with and without the ball. The third focused on the players' attacking decision-making skills with the ball (DMwBall; excluding attacking moments of the game when the player does not have the ball). The final quality concerned decision-making skills without the ball (DMwoBall; when the player's teammate has the ball and is going to attack the opponent's goal).

\section{Statistical analysis}

Analysis of variance (ANOVA) was used to determine if there are any statistically significant differences between the means of the three groups on accuracy and response time. To determine which specific groups differed from each other, post-hoc tests were carried out 
using Tukey correction. Pearson correlations were used to examine the association between performance on the task and coaches opinion.

\section{Results}

There were no statistically significant differences in accuracy between age groups as determined by one-way ANOVA $(P=0.626)$. There was a statistically significant difference between groups as determined by one-way $\operatorname{ANOVA}(F(2,70)=4.960, P=0.010)$. A Tukey post-hoc test revealed that the response time was statistically significantly lower in U23 $(2.03 \pm 0.99 \mathrm{~ms})$ compared to U18 $(2.79 \pm 0.87 \mathrm{~ms}, P=0.018)$ and U16 $(2.75 \pm 0.99 \mathrm{~ms}$, $P=0.030)$. There was no statistically significant difference between the U16 and the U18 groups $(P=0.986)$.

\section{Correlation between performance on the task and coaches opinion in all participants}

Combining all subjects, accuracy on the task was correlated with GeneralDM $\left(r_{s}(73)=0.546\right)$, with Attacking DM $\left(r_{s}(73)=0.576\right)$, with DMwBall $\left(r_{s}(73)=0.554\right)$ and with DMwoBall $\left(r_{s}(73)=0.538\right)$, all for $P<0.001$. On the other hand, reaction time was negatively correlated with DMwoBall $\left(r_{s}(73)=-0.308, P=0.008\right)$.

GeneralDM was correlated with AttackingDM $\left(r_{s}(73)=0.845\right)$, with DMwBall $\left(r_{s}(73)=0.851\right)$, and with DMwoBall $\left(r_{s}(73)=0.794\right)$. AttackingDM was correlated with DMwBall $\left(r_{s}(73)=0.873\right)$ and DMwoBall $\left(r_{s}(73)=0.735\right)$. DMwBall was also correlated with DMwoBall $\left(r_{s}(73)=0.694\right)$, all for $P<0.001$.

\section{Correlation between performance on the task and coaches opinion in the U16 group}

For U16 group, reaction time was negatively correlated with DMwoBall $\left(r_{s}(23)=-0.446\right.$, $P=0.033)$. There were no significant correlations of accuracy measures on the task and the coaches ratings. GeneralDM was correlated with AttackingDM $\left(r_{s}(23)=0.573, P=0.004\right)$, with DMwBall $\left(r_{s}(23)=0.628, P<0.001\right)$, and with DMwoBall $(\rho=0.504, P=0.014)$. AttackingDM was also correlated with DMwBall $\left(r_{s}(23)=0.712, P<0.001\right)$.

\section{Correlation between performance on the task and coaches opinion in the U18 group}

For U18 group, accuracy was correlated with GeneralDM $\left(r_{s}(24)=0.632\right)$, with Attacking $\mathrm{DM}\left(r_{s}(24)=0.678\right)$, with DMwBall $\left(r_{s}(24)=0.576\right)$ and with DMwoBall $\left(r_{s}(24)=0.698\right)$, all for $P<0.001$. There were no statistically significant correlations for any of the coaches ratings and reaction times. GeneralDM was correlated with AttackingDM $\left(r_{s}(24)=0.901\right)$, with DMwBall $\left(r_{s}(24)=0.904\right)$, and DMwoBall $\left(r_{s}(24)=0.888\right)$, all for $P<0.001$. AttackingDM was also correlated with DMwBall $\left(r_{s}(24)=0.902\right)$ and with DMwoBall $\left(r_{s}(24)=0.851\right)$, both for $P<0.001$. Finally, DMwBall was correlated with DMwoBall $\left(r_{s}(24)=0.791, P<0.001\right)$.

\section{Correlation between performance on the task and coaches opinion in the U23 group}

For U23 group, accuracy was correlated with GeneralDM $\left(r_{s}(26)=0.638\right)$, with Attacking $\mathrm{DM}\left(r_{s}(26)=0.737\right)$, with DMwBall $\left(r_{s}(26)=0.678\right)$ and with DMwoBall $\left(r_{s}(26)=0.621\right)$, all for $P<0.001$. There were no statistically significant correlations for any of the coaches ratings and reaction times. GeneralDM was correlated with AttackingDM $\left(r_{s}(26)=0.923\right)$, 
with DMwBall $\left(r_{s}(26)=0.888\right)$, and with DMwoBall $\left(r_{s}(26)=0.865\right)$, all for $P<0.001$. AttackingDM was also correlated with DMwBall $\left(r_{s}(26)=0.903\right)$ and with DMwoBall $\left(r_{s}(26)=0.836\right)$, both for $P<0.001$. Finally, DMwBall was correlated with DMwoBall $\left(r_{s}(26)=0.767, P<0.001\right)$.

\section{Discussion}

The aim of this experiment was to investigate the decision-making skills of different age groups (Under 16, 18 and 23) of elite academy footballers on a video-based task of real-life football scenarios. It also explored the relationship between individual performance on the task and the performance of the footballers on the pitch, as rated by three independent expert football coaches. This allowed us to examine whether this task is useful in predicting realworld decision-making skills. The results show that there was a significant difference in response times between response time was statistically significantly lower in U23 compared to U18 and U16 and there was no statistically significant difference between the U16 and the U18 groups, but no significant difference between age groups on the accuracy of response.

These results have several implications for the way we think about the development of expertise in academy footballers. First of all, considering there were no differences between age group and accuracy on the task. Perhaps, we should not be too surprised about this. If one compares this with visual search results, there are rarely any differences in accuracy on the task. There are often differences in response time because accuracy is not as sensitive as response time. Secondly, considering that decision-making is a skill, one might consider thinking of decision-making in the same way as we think of a developing musician playing the scales. An academy musician will not make mistakes on their scales, but they will not be able to play them as fast as an expert (Woody, 2001).

In terms of response times on the task there were significant differences across the age groups and thus this measure is more informative. What it suggests is that there is a development critical period in football decision-making somewhere between the ages of 18 and 23 (of course we refer to the group averages here, and there will always be an outlier player). This allows us to give recommendations to the coaches about what to expect from 16 and 18-year-olds. Perhaps, we should not be looking at how quickly they play the game, but in developing their decision-making skills, at a slower level. This might be an argument for not pushing a player in whom one has confidence, but actually holding them back and playing him at a lower level so he has time to develop the accuracy and practice the speed. That is a counterintuitive recommendation as this is about what a player can do, not about what he cannot do.

In terms of talent identification, we think of footballers as an early developing game, as opposed to rugby which is a late-developing game. This result suggests that we might be cutting off too early by measuring their decision-making skills at U16 and U18 and generating a self-fulfilling prophecy.

When examining coaches' ratings of general decision-making with accuracy on the task (for all age groups) there was a significant positive correlation. This is an encouraging result as this suggests that the coaches have got a good insight into what the players can actually do. In comparison, there was no significant correlation for response times on the task and ratings of general decision-making across all subjects. It may be that coaches are not sensitive to reaction times as they do not take note that decision-making happens a few seconds before the execution. So, what they usually consider as the "speed of decisionmaking" is the players' execution speed.

In regards to no statistically significant correlations between U16 performance on the task and coach's ratings, what seems to be happening here while the players are given a 
standard decision-making task to do, coaches have been asked to make judgments on players and, of course, these players are not standardized. The next stage of this kind of work is to ask how can we standardized the examples given to the coaches. From these data, it seems that there is a lot of noise in the coaches' opinions because it is not being possible to control for the preconceptions about the players.

However, the positive correlation between performance on the task and coaches opinion on U18 and U23 shows there is accurate knowledge in the training of academy footballers just as we can see reaction times get better with U23, we can see that accuracy also improves across the age groups and that as the players become closer to expertise and there is less noise, the coaches decision become more reliable.

The interesting point to emphasize is that we have a split between information from the scientific data and information from coaching data. The scientific data is revealing that there is information in the timing information which is not being accessed by the coaches, whereas the information from the coaches is telling us that accuracy is easier for them to assess.

Of course, it is important to remember that elite sports are not about groups, it is about the outliers. While, this does not undermine the results here, it reflects the constant conflict in sport science which relates to how to do good science examining group level difference which is how psychology has developed versus how to be sensitive to the individuals' differences which make athletes unique.

\section{References}

Abernethy, M.A., Chua, W.F., 1996. A field study of control system "redesign": the impact of institutional processes on strategic choice. Contemp. Account. Res. 13 (2), 569606.

Abernethy, B., Baker, J., Cote, J., 2005. Transfer of pattern recall skills may contribute to the development of sport expertise. Appl. Cogn. Psychol. 19 (6), 705-718.

Abernethy, B., Neal, R.J., Koning, P., 1994. Visual-perceptual and cognitive differences between expert, intermediate, and novice snooker players. Appl. Cogn. Psychol. 8 (3), $185-211$.

Aglioti, S.M., Cesari, P., Romani, M., Urgesi, C., 2008. Action anticipation and motor resonance in elite basketball players. Nat. Neurosci. 11 (9), 1109.

Baker, J., Cote, J., Abernethy, B., 2003. Learning from the experts: practice activities of expert decision makers in sport. Res. Q. Exerc. Sport 74 (3), 342-347.

Berry, J., Abernethy, B., $\mathrm{C}^{\wedge}$ ot $\quad$ e, J., 2008. The contribution of structured activity and deliberate play to the development of expert perceptual and decision-making skill. J. Sport Exerc. Psychol. 30 (6), 685-708.

Bruce, L., Farrow, D., Raynor, A., Mann, D., 2012. But I can't pass that far! The influence of motor skill on decision making. Psychol. Sport Exerc. 13 (2), 152-161.

Buszard, T., Farrow, D., Kemp, J., 2013. Examining the influence of acute instructional approaches on the decision-making performance of experienced team field sport players. J. Sports Sci. 31 (3), 238-247.

Carling, C., Le Gall, F., Reilly, T., Williams, A.M., 2009. Do anthropometric and fitness characteristics vary according to birth date distribution in elite youth academy soccer players? Scand. J. Med. Sci. Sports 19 (1), 3-9.

Davids, K., Lees, A., Burwitz, L., 2000. Understanding and measuring coordination and control in kicking skills in soccer: implications for talent identification and skill acquisition. J. Sports Sci. 18 (9), 703-714. 
De Waelle, S., Bennett, S., Lenoir, M., Deconinck, F., 2019. Perceptual-Cognitive and Cognitive Skills in Youth Volleyball Players. In: [Poster]. 24th European Conference of Sport Science, 3-6 July, Prague, Czech Republic.

Elsworthy, N., Burke, D., Dascombe, J.B., 2014. Factors relating to the decision-making performance of Australian football officials. Int. J. Perform. Anal. Sport 14 (2), 401410.

Ericsson, K.A., 1996. The acquisition of expert performance: an introduction to some of the issues. In: Ericsson, K.A. (Ed.), The Road to Excellence: The Acquisition of Expert Performance in the Arts and Sciences, Sports, and Games. Lawrence Erlbaum Associates, Inc., pp. 1-50.

Ericsson, K.A., Smith, J. (Eds.), 1991. Toward a General Theory of Expertise: Prospects and Limits. Cambridge University Press.

Ericsson, K.A., Hoffman, R.R., Kozbelt, A., Williams, A.M. (Eds.), 2018. The Cambridge Handbook of Expertise and Expert Performance. Cambridge University Press. 10 Assessing decision-making in elite academy footballers

FourFourTwo, 2018. Revealed! The 15 most Productive Academies In European Football, FourFourTwo, viewed 30 October 2018. https://www.fourfourtwo.com/gallery/revealed-15-most-productive-academieseuropean-football.

Gilis, B., Helsen, W., Catteeuw, P., Wagemans, J., 2008. Offside decisions by expert assistant referees in association football: perception and recall of spatial positions in complex dynamic events. J. Exp. Psychol. Appl. 14 (1), 21.

Hepler, T.J., Feltz, D.L., 2012. Take the first heuristic, self-efficacy, and decision-making in sport. J. Exp. Psychol. Appl. 18 (2), 154.

Huijgen, B.C., Leemhuis, S., Kok, N.M., Verburgh, L., Oosterlaan, J., Elferink-Gemser, M.T., Visscher, C., 2015. Cognitive functions in elite and sub-elite youth soccer players aged 13 to 17 years. PLoS One 10 (12), e0144580.

Johnson, J.G., Raab, M., 2003. Take the first: option-generation and resulting choices. Organ. Behav. Hum. Decis. Process. 91 (2), 215-229.

Kittel, A., Larkin, P., Elsworthy, N., Spittle, M., 2019. Video-based testing in sporting officials: a systematic review. Psychol. Sport Exerc.

Lorains, M., Ball, K., MacMahon, C., 2013. Expertise differences in a video decision-making task: speed influences on performance. Psychol. Sport Exerc. 14, 293-297.

MacMahon, C., McPherson, S.L., 2009. Knowledge base as a mechanism for perceptual cognitive tasks: skill is in the details!. Int. J. Sport Psychol. 40 (4), 565-579.

MacMahon, C., Starkes, J., Deakin, J., 2007. Referee decision making in a video-based infraction detection task: application and training considerations. Int. J. Sports Sci. Coach. 2 (3), 257-265.

Makdissi, M., Davis, G., 2016. The reliability and validity of video analysis for the assessment of the clinical signs of concussion in Australian football. J. Sci. Med. Sport 19 (10), 859-863.

Mann, D.T., Williams, A.M., Ward, P., Janelle, C.M., 2007. Perceptual-cognitive expertise in sport: a meta-analysis. J. Sport Exerc. Psychol. 29 (4), 457-478.

Marteniuk, R.G, 1976. Information Processing in Motor Skills. Holt, Rinehart and Winston.

Meylan, C., Cronin, J., Oliver, J., Hughes, M., 2010. Talent identification in soccer: the role of maturity status on physical, physiological and technical characteristics. Int. J. Sports Sci. Coach. 5 (4), 571-592.

Morris, T., 2000. Psychological characteristics and talent identification in soccer. J. Sports Sci. 18 (9), 715-726. 
Mujika, I., Santisteban, J., Impellizzeri, F.M., Castagna, C., 2009. Fitness determinants of success in men's and women's football. J. Sports Sci. 27 (2), 107-114.

Murr, D., Feichtinger, P., Larkin, P., O 'Connor, D., H€oner, O., 2018a. Psychological talent predictors in youth soccer: a systematic review of the prognostic relevance of psychomotor, perceptual-cognitive and personality-related factors. PloS One 13 (10), p.e0205337.

Murr, D., Raabe, J., Honer, O., 2018b. The prognostic value of physiological and physical characteristics in youth soccer: a systematic review. Eur. J. Sport Sci 18 (1), 62-74.

Roberts, R.E., Anderson, E.J., Husain, M., 2010. Expert cognitive control and individual differences associated with frontal and parietal white matter microstructure. J. Neurosci. 30 (50), 17063-17067.

Roberts, R.E., Bain, P.G., Day, B.L., Husain, M., 2012. Individual differences in expert motor coordination associated with white matter microstructure in the cerebellum. Cereb. Cortex 23 (10), 2282-2292.

Salimin, N., Jani, J., Shahril, M.I., Elumalai, G., 2015. Validity and reliability of comprehensive assessment instruments for handball and badminton games in physical education. Asian Soc. Sci. 11 (23), 12.

Starkes, J.L., Ericsson, K.A., 2003. Expert Performance in Sports: Advances in Research on Sport Expertise. Human Kinetics.

Starkes, J.L., Lindley, S., 1994. Can we hasten expertise by video simulations? Quest 46 (2), 211-222.

Tallir, I., Musch, E., Lanoo, K., Van de Voorde, J., 2003a. Validation of video-based instruments for the assessment of game performance in handball and soccer. In: Proceedings of the 2nd International Conference: Teaching Sport and Physical Education for Understanding, December, 11-14.

Tallir, I., Musch, E., Lenoir, M., Valcke, M., 2003b. Assessment of game play in basketball. In: Conferencia presentada en el 2nd International Conference for Sport and Understanding, Melbourne, December.

Vaeyens, R., Malina, R.M., Janssens, M., Van Renterghem, B., Bourgois, J., Vrijens, J., Philippaerts, R.M., 2006. A multidisciplinary selection model for youth soccer: the Ghent Youth Soccer Project. Br. J. Sports Med. 40 (11), 928-934.

Weigel, P., Raab, M., Wollny, R., 2015. Tactical decision making in team sports-a model of cognitive processes. Int. J. Sports Sci. 5 (4), 128-138.

Williams, A.M., Ward, P., Smeeton, N.J., Allen, D., 2004. Developing anticipation skills in tennis using on-court instruction: perception versus perception and action. J. Appl. Sport Psychol. 16 (4), 350-360.

Wirth, M., Gradl, S., Poimann, D., Schaefke, H., Matlok, J., Koerger, H., Eskofier, B.M., 2018. Assessment of perceptual-cognitive abilities among athletes in virtual environments: exploring interaction concepts for soccer players. In: Proceedings of the 2018 Designing Interactive Systems Conference, June. ACM, pp. 1013-1023.

Woods, C.T., Raynor, A.J., Bruce, L., McDonald, Z., 2016. Discriminating talent-identified junior Australian football players using a video decision-making task. J. Sports Sci. 34 (4), 342-347.

Woody, R.H., 2001. Learning from the experts: applying research in expert performance to music education. Update Appl. Res. Music Educ. 19 (2), 9-14. 